Results Participants included nineteen children. Median age was 7.9 years (range 0.6 years - 18.1 years). Majority were female $(n=14,74 \%)$. Median age at diagnosis was 2.5 weeks (range birth - 2.7 years). Growth Hormone treatment was in place for the majority $(n=14,74 \%)$ commenced at median age of 2.6 years. Of the reporting parents, $89 \%(n=17)$ were mothers, $37 \%(n=7)$ reported to be homemakers. All children were living in 2 parent households.

QOL family impact Families reported 'Worry' about medical treatment, the future, others reactions to their child's condition and impact on other family members as impacting most significantly on the family.

Significant negative correlations between family QOL and age/weight were observed across many domains, the strongest apparent in the area of family functioning and family relationships.

QOL parent proxy reports PWS was perceived by parents to impact most significantly on their child's School functioning. Significant negative correlations with age and weight were observed. Parents identified psychosocial health and social functioning to be most notably affected.

Burden Various areas were identified as significantly adding to caregiver burden such as concerns regarding long-term health and disruptions to family routines.

Conclusion PWS impacts significantly on QOL for the affected child and the entire family.

\section{GP145 TEN MINUTE TALKS - A NOVEL APPROACH TO DELIVERING TEACHING IN A BUSY MEDICAL UNIT}

${ }^{1}$ Jennifer Boyle ${ }^{*},{ }^{2}$ Dominic Cochran, ${ }^{2}$ Neil Patel. ${ }^{1}$ Neonatal unit, Royal Hospital for Children, Glasgow, Glasgow, UK; ${ }^{2}$ Neonatal Unit, Royal Hospital for Children, Glasgow, Glasgow, UK

10.1136/archdischild-2019-epa.209

Introduction It can be challenging to deliver teaching for trainees in busy medical units. One of us (NP) came up with the idea of brief, ten minute teaching sessions following morning handover. The aim was to achieve focused teaching for day and night shift staff in a brief period without impacting upon clinical duties.

Methods Ten minute talks (TMTs) are delivered by consultants on topics of their choosing after morning handover three days a week. The speaker simply talks to the group, usually without visual aids. A kitchen timer is used to alert the group when ten minutes is up ensuring night staff get away promptly and day staff are free to start their duties. Feedback was collated from trainees (anonymously) and consultants to assess success of this new method.

Results/Discussion The development of TMTs has been well received. A wide range of topics have been covered including clinical subjects, items relating to governance and to practical procedures. Trainees have rated TMTs as their favourite method of teaching when compared to more traditional methods such as grand round, journal club and structured 1 hour teaching presentations. On a ten-point scale (1 poor, 10 excellent) they rated TMTs a mean score of 9.7 compared to our other educational activities, which scored $5,8.3$, \& 8 respectively. Similarly, consultants gave a positive review of TMTs rating them 8.5 in terms of effective teaching.

Conclusion We believe TMTs have proved a popular and effective addition to the education programme in our unit and have ensured that focused relevant teaching is delivered thrice weekly to both day and night shift staff in a time pressured environment with a minimum of resources or preparation. We believe this format could easily be adapted to be used in other medical departments to complement their existing teaching.

\section{GP146 THE DEVELOPMENT, IMPLEMENTATION AND EVALUATION OF AN INNOVATIVE NEAR-PEER MENTORING PROGRAMME FOR DOCTORS IN A TERTIARY NEONATAL UNIT}

Bronwyn Power*, Anna Curley. The National Maternity Hospital, Holles Street, Dublin, Ireland

\subsection{6/archdischild-2019-epa.210}

Introduction Near-Peer Mentoring is a validated educational model for personal and professional development ${ }^{1}$. The European Working Time Directive has resulted in reduced, fragmented working hours for Non-Consultant Hospital Doctors (NCHDs) in Ireland. As a result, sustained professional relationships with experienced colleagues may be harder to establish, and mentoring relationships less likely to develop. We aimed to develop and implement a NearPeer Mentoring Programme for NCHDs in a tertiary neonatal unit.

Methods This was a prospective, questionnaire-based, quality improvement initiative. An Education Session on Mentoring was held for all NCHDs working within the unit. Prospective Mentors were asked to meet formally with their Mentees at least four times over a 3-month period. A Mentoring Template was developed to provide a framework for both the Mentors (Registrars/Specialist Registrars) and Mentees (Senior House Officers). This Template was based on four key themes - Paediatric Training in Ireland, Career Development, Professional Skills and Work-Life Balance. Validated, anonymous questionnaires were distributed prior to and following the period of mentorship.

Results All NCHDs wished to be included in the programme, a total of 9 Mentors and 9 Mentees. There was a $100 \%$ response rate to the questionnaires. Three Mentors (33\%) had received prior mentoring training. Prior to participation, eight Mentors (89\%) felt at least moderately skilled in each of the core mentoring skills outlined in the validated Mentor Competency Assessment Tool.

Mentors and Mentees met on average three times over the study period. $100 \%$ of the Mentees and eight of the Mentors (89\%) felt that the programme contributed to a more positive work environment for NCHDs. Seven Mentees (78\%) felt that their clinical skills and knowledge had improved, that their exposure to clinical activities in the NICU was increased, and that a supportive environment was developed. Six Mentees $(67 \%)$ noted an improvement in their procedural skills. Eight (89\%) agreed that their Mentor was accessible and supportive, provided appropriate career guidance and motivated them to reach their objectives.

Conclusion Although mentoring is a core skill specified by the Royal College of Physicians in Ireland for Higher Specialist Trainees in Paediatrics, there is a paucity of formal Mentoring Programmes for NCHDs. This study has highlighted the benefits of a successful Near-Peer Mentorship Programme for Paediatric trainees. 


\section{REFERENCE}

1. Akinla 0 , Hagan $P$, Atiomo W. A systematic review of the literature describing the outcomes of near-peer mentoring programs for first year medical students. BMC Med Educ. 2018;18(1):98.

\section{GP147 LOST IN THE SCENARIO: LOSING SIGHT OF THE PATIENT WHEN USING A STRUCTURED PRESCRIPTION CHART}

Alison Bell*, Tim Dornan, Richard Conn. Queens University Belfast, Belfast, UK

\subsection{6/archdischild-2019-epa.211}

Background Insulin is a dangerous medication and prescribing errors associated with its use can have life-threatening consequences. Holistic prescribing requires involvement of the patient and their family in the process as well as prescribing the medication safely. An insulin prescribing chart structures the task of prescribing insulin but there is a lack of research on its impact on the doctor's behaviour.

Aim To explore how a prescription chart structures the task of prescribing insulin

Methods Qualitative study involving interviews with seven doctors who routinely prescribe insulin for children. Two phase interview: phase one 'free association' interview where doctors described their approach to insulin prescribing; and simulated prescribing task using 'think-aloud' methodology, where doctors verbalised their reasoning while completing an insulin prescription. Interview transcripts were thematically analysed, guided by existing published prescribing frameworks. Doctors' approaches during each interview phase were compared, supported by content analysis of coded data.

Results The introduction of the prescription chart changed the behaviour of doctors while prescribing. Their behaviour moved from a holistic approach to a much narrower, task focused approach to prescribing. Fewer doctors verbalised any intent to interact with the patient while prescribing for them and checked fewer aspects of their prescription when presented with the chart. They moved from a holistic approach to a goal orientated one.

Conclusion This study has shown that the use of a prescribing chart changes doctors' behaviours. Training doctors to use prescribing charts in isolation may de-emphasise the importance of patient engagement. We recommend patient centred prescribing education for medical students and doctors. This may involve a staged progression from prescribing in the classroom setting, to prescribing with a simulated patient, to opportunities to prescribe in the clinical context with supervision.

\section{GP148 DIFFERENCES IN HOSPITAL ADMISSIONS OF REFUGEE AND LOCAL PEDIATRIC POPULATION IN ISRAEL}

\footnotetext{
${ }^{1}$ Michael Schnapper*, 'Avshalom Oziri, 'Adi Ovadia, 'Shirly Abiri, 1,2Diana Tasher, 1,2llan Dalal. 'Pediatric Department, E Wolfson Medical Center, Holon, Israel; ${ }^{2}$ Sackler faculty of medicine, Tel Aviv University, Tel aviv, Israel
}

\subsection{6/archdischild-2019-epa.212}

Background The ongoing refugee crises around the world have raised significant concerns among medical communities worldwide. By the end of 2016, there were 40,274 registered refugees residing in Israel according to immigration authorities. It is estimated that among them, are 5500 children.

Aim To examine and identify the differences between refugee and Israeli children admitted to the pediatric department.

Methods All data relevant to admissions to the pediatric department at 'Wolfson Medical Center' in Israel, between the years 2013-2017 was collected. We than compared the data between two groups; refugees and local Israeli children.

Results During our study, there were 654 refugee and 11,858 Israeli children admitted to the pediatric department from the Pediatric Emergency Department (admissions rate of $28.3 \%$ and $13.8 \%$ respectively); average age of admission was 1.2 among refugees and 4.7 years in the local group $(\mathrm{P}<0.01)$; average duration for a single hospital stay was 3.13 days in the refugee group and 2.49 days in the local group $(\mathrm{P}<0.01)$, with prolonged stay (i.e. longer than 14 days) also found to be significantly different with $2.7 \%$ of all admissions in the refugee group, whereas the control group had $2.3 \%$ prolonged admissions; We further found differences in common diagnoses leading to hospitalization, with leading cause among refugees being skin infections whilst the gastrointestinal system was the predominant cause for admissions in the local group. Important information emerged regarding healthcare coverage. While refugee children had a coverage percentage of $76.76 \%$, among Israeli children health care program coverage was of $98.35 \%$.

Conclusions In this retrospective study we found evidence of significant morbidity in children of refugees as compared to local Israeli pediatric population. This is supported by higher admissions rates, younger age of admission and higher percentage in long duration of stay. Furthermore, there are major differences between the two populations in diagnosis leading to admission. With the ever growing global refugee crises generating an ever growing number of displaced children, these findings should prompt further study in order to benefit refugee children in welcoming communities worldwide.

\section{GP149 INTRODUCING A MINIMUM ACCEPTED COMPETENCY (MAC) EXAM FOR COMMENCING SUPERVISED PAEDIATRIC PRACTICE}

${ }^{1,2}$ Paddy McCrossan*, 'Naomi McCallion. 'Royal College of Surgeons in Ireland, Dublin, Ireland; ${ }^{2}$ Royal College of Physicians in Ireland, Dublin, Ireland

\subsection{6/archdischild-2019-epa.213}

Aims To determine if undergraduate students and paediatric trainee doctors level of knowledge meet a non-faculty clinician-determined minimum accepted competency (MAC).

Methods A 30-item multiple-choice (MCQ) paper (MAC exam) was created, formed of questions proposed by practising non-academic consultant paediatricians, which are deemed as 'must know' for paediatric trainees prior to commencing clinical work.

A 'passing score' was determined using the Angoff technique by the paediatric faculty.

The paper was given to undergraduate students following their formal paediatric teaching and also paediatric senior house officers (SHO's). 